\title{
Entwicklung eines komplexen Führungsverständnisses mit Hilfe von psychodramatischer Organisationsaufstellung
}

\author{
Sylvia Schweiger
}

Angenommen: 22. Januar 2021 / Online publiziert: 10. Februar 2021

(C) Der/die Autor(en) 2021

Zusammenfassung Dieser Artikel der Zeitschrift für Psychodrama und Soziometrie zeigt auf, dass es nicht einfach ist, eine (gute) Führungskraft zu sein. Allerdings hilft es, sich ein komplexes Verständnis von Führung anzueignen. Ein komplexes Verständnis von Führung zu haben impliziert, dass Individuen über die Fähigkeit verfügen, dialektisch zu denken. Die aktuelle Forschung liefert allerdings wenig konkrete Ansätze, wie ein derartiges Denken gefördert werden kann. Mit Hilfe eines Fallbeispiels, in dem die Methode der psychodramatischen Organisationsaufstellung angewandt wurde, leistet dieser Artikel drei konkrete Beiträge. Erstens zeigt er auf, dass die Methode der psychodramatischen Organisationsaufstellung wesentlich dazu beitragen kann, ein komplexes Führungsverständnis zu entwickeln. Zweitens veranschaulicht dieser Artikel, dass durch die Anwendung der psychodramatischen Organisationsaufstellung neue Forschungserkenntnisse generiert werden können. Drittens erörtert der Beitrag, warum die psychodramatische Organisationsaufstellung als eine äußerst vielversprechende Methode zur Datengenerierung und Datenanalyse im Bereich der empirischen Sozialforschung angesehen werden kann.

Schlüsselwörter Psychodrama P Psychodramatische Organisationsaufstellung • Führungskräfteentwicklung · Komplexes Führungsverständnis · Dialektisches Denken · Empirische Sozialforschung 


\title{
Developing some complex leadership meaning with the aid of psychodrama sessions
}

\begin{abstract}
This article shows that being a (good) leader is not easy. However, developing some complex leadership meaning helps. Understanding leadership in a complex way implies that individuals are able to adopt dialectical thinking. Yet, current research keeps being vague about how such thinking can be promoted. Based on a case study, in which the method of psychodrama sessions was applied, this article offers three contributions. First, the article illustrates that the method of psychodrama sessions can substantially contribute to developing some complex leadership understanding. Second, the article shows that the application of psychodrama sessions may support generating new insights. Third, this article considers psychodrama sessions as a most promising method for collecting and analyzing data within empirical social research.
\end{abstract}

Keywords Psychodrama - Psychodrama session · Leadership development · Complex leadership understanding · Dialectical thinking $\cdot$ Empirical social research

\section{Die Entwicklungsstufen eines Führungsverständnisses}

Wer bin ich als Führungskraft? Wer möchte ich als Führungskraft sein? Ist es mir wichtig, dass mich meine MitarbeiterInnen mögen und dass ich ihnen gefalle? Möchte ich andere inspirieren, leiten und motivieren? Wie gehe ich als Führungskraft mit Konflikten um? Was tue ich, wenn MitarbeiterInnen stur ihren eigenen Weg gehen und sich nicht von mir führen lassen? Wie stark ist es meine Aufgabe MitarbeiterInnen zu kontrollieren und Prozesse zu regulieren? Soll ich lieber partizipativ oder autoritär führen? Wie nah darf ich meinen MitarbeiterInnen sein, ohne dabei ihren Respekt zu verlieren? Welche Dynamik herrscht in meinem Team? Wie kann ich die Teamdynamik positiv beeinflussen? Solche oder ähnliche Fragen stellen sich viele Führungskräfte und beantworten sie auf die vielfältigste Art und Weise.

Was man unter Führung verstehen kann, ist wohl so mannigfaltig wie die Menschheit an sich. Ein Blick in die Literatur zeigt, dass die vielfältigen Bedeutungen von Führung in drei Kategorien unterteilt werden können, die spezifischen Entwick-

Tab. 1 Führungsverständnis anhand von drei Entwicklungsstufen (In Anlehnung an Day und Harrison 2007 \& Hammond et al. 2017)

\begin{tabular}{lll}
\hline Entwicklungsstufen & Verständnis von Führung & Analyseebene \\
\hline $\begin{array}{l}\text { Einfaches Füh- } \\
\text { rungsverständnis }\end{array}$ & Führung ist ein top-down Prozess & Individuelle Ebene \\
$\begin{array}{l}\text { Moderates Füh- } \\
\text { rungsverständnis }\end{array}$ & $\begin{array}{l}\text { Führung ist ein reziproker Beeinflussungsprozess (top- } \\
\text { down und bottom up) }\end{array}$ & $\begin{array}{l}\text { Individuelle \& rela- } \\
\text { tionale Ebene }\end{array}$ \\
$\begin{array}{l}\text { Komplexes Füh- } \\
\text { rungsverständnis }\end{array}$ & $\begin{array}{l}\text { Führung ist ein von allen Beteiligten gemeinsam kon- } \\
\text { struiertes Sozialsystem mit Interdependenzen zwischen }\end{array}$ & $\begin{array}{l}\text { Individuelle, rela- } \\
\text { tionale \& kollektive }\end{array}$ \\
& Individuen, Gruppen und Organisationen & Ebene \\
\hline
\end{tabular}


lungsstufen zugeteilt sind (siehe Tab. 1). Demnach verstehen Individuen je nach Entwicklungsstufe etwas anderes davon, was es bedeutet, eine Führungskraft zu sein und verfügen auch über unterschiedliche Analyseebenen.

Ein einfaches Führungsverständnis geht davon aus, dass Führung ein top-down Prozess ist, und suggeriert Planbarkeit und Beherrschbarkeit von Situationen. Demnach gilt es als Führungskraft, Anweisungen zu erteilen, zu kontrollieren und zu regulieren sowie die MitarbeiterInnen mit notwendigen Ressourcen adäquat auszustatten, damit sie ihre Arbeit effizient erledigen können. Kurzum, die Führungskraft beeinflusst ihre MitarbeiterInnen. Dieses Verständnis analysiert Situationen aus einer individuellen Perspektive, d.h. das Verhalten einzelner Individuen wird betrachtet. Der Erfolg von Führung zeichnet sich dadurch aus, dass MitarbeiterInnen die Erwartungen der Führungskraft erfüllen (Müller und Schweiger 2018).

Haben AkteurInnen ein moderates Führungsverständnis, sehen sie Führung als reziproken Beeinflussungsprozess zwischen Führungskraft und MitarbeiterInnen und somit nicht nur als top-down, sondern auch als bottom-up Prozess. Dementsprechend beeinflusst nicht nur die Führungskraft ihre MitarbeiterInnen, sondern MitarbeiterInnen beeinflussen auch ihre Führungskraft. Verfügt man über dieses Führungsverständnis, werden Situationen aus sowohl einer individuellen als auch einer relationalen Perspektive (d.h. Beziehungen zwischen Individuen werden betrachtet) analysiert. Der Erfolg von Führung zeichnet sich dadurch aus, dass Führungskräfte und MitarbeiterInnen gegenseitig ihre an sie gestellten Erwartungen erfüllen (Day und Harrison 2007).

Personen mit einem komplexen Verständnis nehmen Führung als ein von allen Beteiligten gemeinsam konstruiertes soziales System mit eigenen Normen und Werten wahr. Der Fokus verschiebt sich nun auf das Kollektiv, sprich, auf das gesamte Sozialsystem, das aus Individuen, Dyaden, Gruppen, Abteilungen und Organisationen besteht, die sich fortwährend gegenseitig beeinflussen und dadurch gemeinsam ihre Normen und Werte definieren. Um ein System ganzheitlich zu analysieren, bedarf es aller drei Analyseebenen: Wie ergeht es den einzelnen Organisationsmitgliedern (individuelle Ebene), wie ergeht es den Beziehungen zwischen Individuen (relationale Ebene) und wie ergeht es der gesamten Gruppe, Abteilung oder Organisation (kollektive Ebene)? Der Erfolg von Führung zeichnet sich dadurch aus, dass Normen und Werte etabliert sind, die sinnvoll und stimmig für alle Ebenen sind (Hammond et al. 2017; Müller und Schweiger 2018; Schweiger 2019; Schweiger et al. 2020).

\section{Vom dichotomen zum dialektischen Denken}

Je mehr man sein Führungsverständnis entwickelt, desto eher ist man imstande, dichotomes Entweder-oder Denken abzulegen und sich dialektisches Sowohl-alsauch Denken anzueignen. Dialektisches Denken unterstützt dabei, Zusammenhänge, Spannungen, Asymmetrien und Widersprüchlichkeiten, die sich fortlaufend in einem Führungskräftealltag ergeben, nicht nur zu erkennen, sondern auch zu würdigen. Beispielsweise werden in der Leadership Forschung partizipatives und autoritäres Führen oft als zwei widersprüchliche Verhaltensformen gesehen, die sich kaum 
vereinbaren lassen. Ein dichotomes Entweder-oder Denken plädiert dafür, sich für einen der beiden Führungsstile zu entscheiden oder - falls dies zu extrem erscheint beide Verhaltensformen sequentiell, je nach Situation, an den Tag zu legen. Stattdessen versucht dialektisches Denken, die Hintergründe und Motive dieser beiden Stile zu erkennen, um schlussendlich zu einem für das entsprechende Sozialsystem stimmigen und sinnvollen Führungsverhalten zu gelangen (Collinson 2014; Hammond et al. 2017).

Das „WAS“ scheint somit klar dargelegt: Aktuelle Forschung im Bereich Leadership rät Führungskräften sich ein komplexes Führungsverständnis anzueignen und dialektisches Denken zu praktizieren (Schweiger et al. 2020). Allerdings bietet die Literatur kaum nützliche Methoden, „WIE“ man von einem einfachen zu einem komplexen Führungsverständnis gelangt bzw. dialektisches Denken fördern kann. Dieser Artikel zeigt auf, dass die Methode der psychodramatischen Organisationsaufstellung hierbei einen bedeutsamen Beitrag leisten kann.

\section{Die psychodramatische Organisationsaufstellung}

Wissenschaftstheoretische Grundlage der psychodramatischen Organisationsaufstellung nach Moreno stellt die Handlungstheorie dar, die davon ausgeht, dass soziale Wirklichkeit durch Handlungsprozesse konstruiert wird (Buer 2003, S. 44). Menschen begegnen sich, verhalten sich in einer bestimmten - unter Umständen auch überraschenden - Art und Weise und konstruieren dabei gemeinsam ihre Wirklichkeit. Moreno hat davon die Aktionssoziometrie, auch bekannt als soziometrische Netzwerktheorie, abgeleitet (Riepl 2016, S. 200). Demnach können Menschen als Knoten von Beziehungen und Organisationen als Interaktionsnetze gedacht werden. Diese Interaktionsnetze können auf einer formellen Rollenstruktur oder einer informellen Beziehungsmatrix beruhen (Buer 2003, S. 53).

Konflikte und Probleme in Organisationen entstehen mitunter dann, wenn es zu einem „Auseinanderklaffen zwischen der Oberflächenstruktur - den formalen Rollen wie Geschäfts- und Abteilungsleiter, Sachbearbeiter - und der sogenannten sozioemotionalen Tiefenstruktur (Anziehung, Abstoßung, Nähe, Distanz etc.)“ (Riepl 2016, S. 199) kommt. Morenos Ansatz geht davon aus, dass jeder Mensch bestrebt ist, ,in Konstellationen zu leben, die durch ein von ihm bestimmtes Maß an Anziehungen und Abstoßungen gekennzeichnet ist" (Buer 2003, S. 43). Je näher sich der Mensch an seinem Idealzustand befindet, desto zufriedener, gesünder, produktiver und kreativer sei er (Buer 2003, S. 43). Davon kann für Organisationen abgeleitet werden, dass je konsistenter und balancierter die formellen oberflächlichen Rollenbeziehungen und die informellen sozioemotionalen Bindungswünsche auf der Tiefenebene sind, desto zufriedener, gesünder, produktiver und kreativer sind die Organisationsmitglieder.

Die psychodramatische Organisationsaufstellung bietet die Möglichkeit, unterschwellige sozioemotionale Netze bzw. Konfigurationen (wie Paare, Dreiecke, Ketten, Stars, Isolierte), Verfestigungs- und Flexibilisierungstendenzen, Nähe und Distanz, positive und negative Beziehungen sowie Bindungswünsche von Organisationsmitgliedern sichtbar und dadurch veränderbar zu machen (Buer 2003, S. 47). 
Dies wird ermöglicht, indem eine surplus reality geschaffen wird (Von Ameln und Kramer 2007, S. 18). Eine surplus reality bildet nicht „die Realität“ ab, sondern versinnbildlicht momentane Gedanken und Gefühle der ProtagonistInnen. Somit ist diese Darstellung immanent subjektiv und verkürzt. Und trotzdem stellt eine surplus reality einen symbolischen Erlebensraum dar, der zu neuen Erkenntnissen und verstärktem Empowerment der ProtagonistInnen führen soll (Riepl 2016, S. 206).

Die psychodramatische Organisationsaufstellung gliedert sich in die drei Phasen (a) Erwärmungsphase, (b) Aufstellungsphase und (c) Transferphase. In der (a) Erwärmungsphase werden mitunter das übergeordnete Managementthema, die aktuell zu bearbeitende Aufstellungsfrage und die Ziele zur Aufstellungsfrage festgehalten. Zudem muss geklärt werden, in welcher Form aufgestellt wird. In Einzelsettings (egozentrierte Vorgangsweise) kann man ein Ressourcogramm anfertigen, eine Aufstellung mit Stühlen durchführen oder ein Systembrett mit Klötzchen bzw. Symbolen wie Holztieren verwenden. In Gruppensettings (gruppenzentrierte Vorgangsweise) unterscheidet man zwischen stranger groups (Einzelpersonen gehen mit Menschen, die ihnen fremd sind, einer berufsbezogenen Frage nach) und organisationsinternen Gruppen, wobei hier nochmals zwischen kontinuierlichen Teams und vorübergehenden Projektteams unterschieden wird (Riepl 2016, S. 200-201).

In der (b) Aufstellungsphase wird zunächst von der Protagonistin bzw. vom Protagonisten ein erstes Bild (vergangene Konstellation oder Ist-Zustand) aufgestellt und danach Prozessarbeit (Soll-Zustand) geleistet. Dabei pendelt die Aufstellungsarbeit zwischen dem Einnehmen einer Außenperspektive durch die Spiegelposition und dem Einnehmen einer Innensicht durch den Rollenwechsel. Die Spiegelposition verschafft einen Überblick und ,bringt den Thementräger durch einen Perspektivenwechsel in eine Quasi-Beobachterrolle dem aufgestellten System gegenüber“" (Von Ameln und Kramer 2007, S. 256). Der Rollenwechsel hingegen findet im Aufstellungsbild statt. Dabei übernimmt der oder die ProtagonistIn die Rolle einer aufgestellten Person. Dies ermöglicht ein Wahrnehmen, wie eine bestimmte Person in Beziehung zu den anderen Personen steht (Buer 2003, S. 47-48; Riepl 2016, S. 205). Der oder die AufstellungsleiterIn hat dabei die Aufgabe, den Prozess zu begleiten, indem er bzw. sie Regie führt, spontan steuert, geduldig wartet bis eine Einsicht aufscheint und am richtigen Punkt schließt. „Einsichten sollten die Chance haben, zu gegebener Zeit aufzutauchen. Dann sind sie am treffendsten und am wirksamsten“ (Buer 2003, S. 47). Der oder die LeiterIn deutet bzw. bewertet nicht und macht keine Vorschläge. Somit ist der Prozess nicht leitungszentriert, sondern egozentriert bzw. gruppenzentriert (Buer 2003). „Es werden keine Lösungen angepeilt, sondern Aufgaben, und die sich dabei konkret organisierenden Personen werden in den Mittelpunkt der Beratung gestellt. Unaufhörlich geht es dabei um die Sichtweisen dieser Personen und deren unterschiedliche Einschätzungen von Organisationsthemen“" (Riepl 2016, S. 199).

In der (c) Transferphase führen AufstellungsleiterIn und ProtagonistIn das Aufstellungsbild an das Managementthema, die Aufstellungsfrage und die Ziele zur Aufstellung zurück. Hierbei soll zum Handeln motiviert und an einer konkreten Umsetzung gearbeitet werden, wobei Vor- und Nachteile bzw. Aufwand und Nutzen von geplanten Veränderungen sowie auch ethische Aspekte besprochen werden können (Buer 2003; Riepl 2016, S. 211). 


\section{Das Fallbeispiel „Vom Teammitglied zum Abteilungsleiter“}

Dieses Kapitel widmet sich nun einer Fallstudie zum Managementthema „Vom Teammitglied zum Abteilungsleiter". Diese Studie wurde mit Hilfe der Methode der psychodramatischen Organisationsaufstellung im Jahr 2018 durchgeführt. Zum Hintergrund des Protagonisten ist zu erwähnen, dass er mehrere Jahre Teammitglied in seiner Abteilung war, bevor er 2015 zum Abteilungsleiter in einem mittleren Unternehmen ernannt wurde. 2018 leitet er 13 MitarbeiterInnen. Seinem Empfinden nach könnte es in der Abteilung insgesamt ,runder laufen“. Er habe des Öfteren das Gefühl, dass er die Kontrolle und den Überblick verliere. Anstatt sich einer langfristigen strategischen Neuausrichtung zu widmen, müsse er sehr oft das „Feuer“ bei kurzfristigen Projekt-Brandherden löschen. Außerdem hätten vor kurzem zwei Mitarbeiter gekündigt. Davon abgeleitet formuliert der Protagonist folgende Aufstellungsfrage: „Wie sieht die Teamdynamik in meiner Abteilung aus?“. Das Ziel dieser psychodramatischen Organisationsaufstellung ist es, einen besseren Überblick über die Teamdynamik in der Abteilung zu erhalten und davon adäquate nächste Schritte abzuleiten, um die Teamdynamik positiv zu beeinflussen.

\subsection{Beschreibung der Methode}

In diesem Einzelsetting kommt das Systembrett mit Klötzchen zur Anwendung. Zunächst bitte ich den Protagonisten, das Team im Jahr 2015 - das Jahr in dem er Abteilungsleiter wurde - aus seiner heutigen Sicht aufzustellen. Nach einem Rollenwechsel und dem Einnehmen der Spiegelposition, stellt der Protagonist sein Team im Jahr 2018 auf das Brett. Hierauf folgen eine Beschreibung des Bildes aus diversen Rollenwechsel und vier Spiegelpositionen nach jeweils 90 Grad Drehungen des Systembretts. In der Spiegelposition stelle ich als Aufstellungsleiterin folgende Fragen: „Sehen Sie nach dieser 90 Grad Drehung etwas, das Sie vorher nicht gesehen haben? Was sagt Ihnen das? Ist das repräsentativ für Ihre gegenwärtige berufliche Situation? Darf ich Sie bitten aufzustehen, um sich das aus dieser Position anzuschauen? Was würde Ihnen ein guter Freund raten, wenn er das sieht?“. Während des Rollenwechsels stelle ich unter anderem Fragen wie: „Wenn Sie den Klötzchen in die Augen schauen, was für eine Qualität hat dieser Blick? Wie viel Sympathie herrscht hier, wie viel Respekt herrscht hier? Haben Sie einen Impuls? Was würden Sie gern machen? Beschreiben Sie gerade den Ist-Zustand oder Ihren Wunsch aus dieser Perspektive?“

\subsection{Beschreibung der surplus reality}

In der surplus reality vom Jahr 2015 sieht sich der Protagonist mit der Frage konfrontiert, wie nah er seinen MitarbeiterInnen sein darf bzw. wie distanziert er ihnen gegenüber eigentlich sein sollte. Der Abteilungsleiter beschreibt dabei eine Entweder-oder Situation: entweder stelle er sich auf die Seite seiner MitarbeiterInnen oder auf die Seite des Geschäftsführers. ,Das sind jetzt die großen Herausforderungen: die Positionsbestimmung, die eigene Position. In welche Richtung soll die Bewegung gehen? Wo ist die Nähe? Die Nähe zu welchen anderen Teilnehmern soll gesucht 
werden? Ist es wichtiger, in die Richtung der Geschäftsführung zu wandern? Ist es wichtiger, möglichst da zu bleiben bei den Projektleitern in diesem Verbund? Oder ist es wichtiger, den Weg zum Team zu suchen? (...) Wenn ich mich umdrehe und zur Geschäftsführung schaue, dann sehe ich weder Team noch die Projektleiter. Das ist schwierig, da die Position zu finden. Man kann natürlich noch weiter nach außen gehen, dann sieht man zwar alles, aber dann hat man eine irrsinnige Distanz. Dann verliert man die Nähe, aber man überblickt die Situation. Je nachdem in welche Richtung man wandert, man hat immer irgendwo den Nachteil, dass man entweder den Fokus verliert oder eine Nähe zu irgendjemanden. Das ist immer so eine Frage, welches Trade-off man eingehen möchte“.

Nach dem Aufstellen der surplus reality vom Jahr 2018 beschreibt der Protagonist, dass er sich im Laufe der Zeit von den MitarbeiterInnen weg in Richtung Geschäftsführung bewegt hat. „Ich finde die Mitarbeiter eben sympathisch und möchte auch gern in die Umgebung dieser Personen. Aber das bedeutet, dass man zu anderen Personen dann einen größeren Abstand bekommt, was auch wieder nicht Ziel sein soll. Insofern ist das sozusagen ein bisschen eine Schwierigkeit oder eine schwierige Aufgabe, da diese eigene Position zu finden, auf dem Spielbrett. Weil man sich verbunden fühlt mit denen, möchte ich näher kommen. Auf der anderen Seite ist dann aber auch im Hinterkopf diese Idee, dass das auch mit einem Nachteil verbunden ist und dass man es daher besser so belässt, wie es ist. Dass das schon seinen Sinn hat, dass da eben in diese Richtung eine größere Distanz ist. (...) Je näher ich den Mitarbeitern komme, desto enger ist der Fokus und desto weniger Überblick habe ich. Insofern ist es besser, den nötigen Abstand zu haben bzw. zu halten.“

Somit lässt sich festhalten, dass der Protagonist in der Aufstellung von 2018 nach wie vor dieselbe Entweder-oder Perspektive einnimmt: entweder stellt er sich auf die Seite seiner MitarbeiterInnen oder auf jene des Geschäftsführers. In den letzten Jahren ist diese Entscheidung zugunsten der Nähe zum Geschäftsführer ausgefallen. Richtig glücklich sei der Protagonist mit dieser Situation nicht, wenngleich er argumentiert, dass dieser Weg wohl einfach notwendig und vernünftig sei, um als Abteilungsleiter einen guten Überblick zu bewahren.

Als Nächstes bitte ich den Protagonisten, seinen Soll-Zustand auf das Systembrett zu stellen. Als ersten Schritt holt er all seine MitarbeiterInnen näher an sich heran. Dann tauscht er sein eigenes Klötzchen gegen ein Klötzchen aus, das der Größe des Geschäftsführers entspricht. Damit ist der Protagonist aber nicht zufrieden und stellt das zuvor verwendete mittelgroße Klötzchen wieder auf das Brett zurück. „Ein anderer Schmäh wäre dann natürlich, dass ich sage, ich mache es von oben, dann begebe ich mich aber überhaupt auf eine ganz andere Ebene, was ich aus dem Bauch raus als ganz eigenartig empfinde. Insofern möchte ich trotzdem auf dem Boden der Tatsachen bleiben“.

Nach einem kurzen Moment des Zögerns kommt der Protagonist in eine Spontanitätslage und greift nach seinem Autoschlüssel. Diesen Autoschlüssel platziert er vorsichtig unter das mittelgroße Klötzchen, sodass die Größe des Abteilungsleiters nun genau zwischen den MitarbeiterInnen und dem Geschäftsführer liegt. Der Protagonist fährt fort: „Das wäre natürlich eine Idee. (...) Das würde natürlich den Blick ermöglichen, man kann die Abstände beibehalten und trotzdem hat man es dann geschafft, dass die Projektleitung zur Mitte kommt und der Blick trotzdem gewahrt 
bleibt. Für die Mitarbeiter ist dieser Unterschied jetzt klarer. So eine Zwischengröße würde schon gut passen“.

Daraufhin habe ich die Frage gestellt, ob jetzt geklärt sei, wohin die Reise nun gehe. Der Protagonist antwortet: „Nach oben!“

Nach der gewonnenen Erkenntnis, dass die Reise für den Abteilungsleiter nach oben gehen muss, um eine für alle Beteiligten bestmögliche Situation zu schaffen, beginnt die Transferphase. Als nächsten Schritt sieht der Protagonist, dass seine Rolle als Abteilungsleiter gemeinsam mit dem Geschäftsführer ganz klar definiert werden soll. ,Der Autoschlüssel, mein Podest, dieses Fundament soll für mich definiert werden. Das ist das, was mir total wichtig ist".

Zusätzlich zu dieser klaren Rollendefinition will der Protagonist darauf Wert legen, behutsam und in Ruhe die nächsten Schritte zu gehen. Er möchte Energie tanken und sich bewusst Zeit nehmen, um gute Entscheidungen treffen zu können. Als Symbol für das Energie tanken, die Ruhe und das sich Zeit nehmen steht der Schal in Form eines roten Bandes, das der Protagonist zum Abschluss dem Klötzchen, das ihn selbst repräsentiert, umbindet.

Zusammengefasst lässt sich festhalten, dass es dem Protagonisten im Laufe der psychodramatischen Organisationsaufstellung gelingt, ein Entweder-oder Denken abzulegen und eine Sowohl-als-auch Perspektive einzunehmen. Indem der Abteilungsleiter seine hierarchisch höher gestellte formale Rolle auch tatsächlich annimmt und beginnt, Entscheidungen zu treffen und Verantwortung zu übernehmen, kann er seinen MitarbeiterInnen trotzdem nahe sein. Der Protagonist erkennt, dass sich Führung von und Nähe zu MitarbeiterInnen nicht gegenseitig ausschließen. Mit Hilfe dieses Fallbeispiels illustriert dieser Artikel drei konkrete Beiträge der psychodramatischen Organisationsaufstellung zur Sozialforschung, die nun abschließend dargestellt werden.

\section{Drei Beiträge der psychodramatischen Organisationsaufstellung}

\subsection{Beitrag 1: Führungskräfteentwicklung unterstützen}

Der Prozess, generell als Führungskraft zu reifen und damit einhergehend sein Führungsverständnis zu entwickeln, stellt besonders für Nachwuchsführungskräfte eine große Herausforderung dar, auf die sie oft nicht vorbereitet sind und die nicht selten mit Unsicherheit und Unbehagen einhergeht. „Führungskräfte, vor allem junge Nachwuchsführungskräfte, sind (...) in der Verantwortung, Teams oder ganze Unternehmen zu leiten, befinden sich in machtvollen Positionen, sollen ihre MitarbeiterInnen motivieren und gleichzeitig Unternehmensziele erreichen. Solche Situationen sind komplex, mehrdeutig, dynamisch, konfliktbehaftet und damit unsicher" (Müller und Schweiger 2018, S. 55). Gerade diese Unsicherheit untergräbt oft Lernen und Entwicklung und führt dazu, ideologisierten HeldInnenbildern nachzueifern, simplen Weisheiten zu folgen und dichotomes Schwarz-Weiß Denken anzuwenden. Stattdessen gilt es, dieser Unsicherheit entgegenzuwirken und eine differenziertere Perspektive auf sich selbst, die eigenen Leistungen, das eigene Team und dessen Leistungen einzunehmen und damit Führung im sozialen Zusammenhang wahrzu- 
nehmen (Schweiger et al. 2020). Wie das Fallbeispiel zeigt, ist die psychodramatische Organisationsaufstellung eine äußerst hilfreiche Methode, die Komplexität von Führung darzustellen und folglich in einer eigenständigen Art und Weise Ordnung in dieser Wirklichkeitskonstruktion zu schaffen. Auf Basis von Ordnung und Klarheit können ProtagonistInnen schließlich ihrer Unsicherheit entgegenwirken und ein komplexes und gleichzeitig maßgeschneidertes Verständnis von Führung entwickeln.

\subsection{Beitrag 2: Neue Forschungserkenntnisse generieren}

Aktuelle Leadership Forschung ist sich uneinig darüber, wie viel Nähe Führungskräfte zu ihren MitarbeiterInnen aufbauen sollen. Zum einen gibt es Studien, die die Wichtigkeit des Miteinanders, der Partizipation, des Kollektivs sowie der gegenseitigen Sympathie und Wertschätzung betonen (Collinson et al. 2018). Andere Studien wiederum sprechen sich für die Notwendigkeit aus, dass Führungskräfte manchmal unangenehme Entscheidungen treffen und somit auf MitarbeiterInnen Einfluss nehmen müssen, auch wenn sich letztere damit wenig einverstanden zeigen (Alvesson und Jonsson 2018). Die Literatur spricht hierbei von einem ,,acting on others“ versus ,acting with others“" Dilemma (Schweiger 2019).

Das oben angeführte Fallbeispiel zeigt, wie dieses Dilemma aufgehoben werden kann, indem es veranschaulicht, dass sich Führung von MitarbeiterInnen (acting on others) und Nähe zu MitarbeiterInnen (acting with others) nicht gegenseitig ausschließen müssen. Allerdings bedarf es für die Integration beider Verhaltensformen nicht nur eine Rollenklarheit (d.h. welche Verantwortung bzw. Erwartungshaltung geht mit welcher Rolle einher?) für alle Teammitglieder, sondern auch eine klare Rollendifferenzierung (d.h. wie unterscheiden sich die unterschiedlichen Rollen voneinander?) zwischen der Führungskraft und den MitarbeiterInnen. Im Soll-Zustand unterscheidet sich das Klötzchen des Abteilungsleiters sowohl in Größe als auch in Farbe von den MitarbeiterInnen-Klötzchen. Somit können wir durch diese Fallstudie die Erkenntnis generieren, dass erst das Schaffen einer Klarheit über die Andersartigkeit ein konstruktives Miteinander ermöglicht.

\subsection{Beitrag 3: Methodenkoffer der empirischen Sozialforschung erweitern}

Durch die psychodramatische Organisationsaufstellung kann der Methodenkoffer der empirischen Sozialforschung um eine äußerst vielversprechende Methode zur Datensammlung und Datenanalyse erweitert werden. Im Gegensatz zu üblichen Methoden, wie Interviews oder Fokusgruppen, stellt die psychodramatische Organisationsaufstellung eine neue Form der Kommunikation dar, die die Wirklichkeitskonstruktionen der ProtagonistInnen expliziert. Dabei spricht diese Methode alle drei Analyseebenen an. Die (1) individuelle Ebene wird durch die dargestellten Charakteristika der Individuen veranschaulicht. Beispielsweise haben Klötzchen auf einem Systembrett eine bestimmte Größe, Rundung, Farbe und Dicke. Zudem ermöglicht diese Methode einen Rollenwechsel und kann somit auch Daten zu konstruierten Fremdansichten zur eigenen Person, zu anderen Personen und zur Gesamtsituation sammeln. Die (2) relationale Analyseebene wird angesprochen, indem die Organisationsaufstellung zeigt, wie Individuen zueinanderstehen: Schauen sie sich an 
oder kehren sie einander den Rücken zu? Wie groß ist der Abstand? Welches Klötzchen ist größer/kleiner, dicker/dünner, rund/eckig oder hat eine gleiche/andere Farbe im Vergleich zu anderen Klötzchen? Damit werden Status- und Beziehungsmuster dargestellt. Die (3) kollektive Analyseebene findet sich durch die Betrachtung der gesamten surplus reality Bühne wieder. Dadurch können kollektive Muster erkannt werden, die auf Normen und Werte der sozialen Gruppe schließen lassen. Des Weiteren bildet diese Methode nicht nur bereits vorhandene Wirklichkeitskonstruktionen ab, sondern kann - wie oben gezeigt - auch dabei unterstützen, neue Erkenntnisse zu generieren. Eine klare Trennung zwischen Ist-Zustand und Soll-Zustand sowie eine sich wiederholende Abfolge von Spiegelposition und Rollenwechsel - also ein ständiger Wechsel der Perspektiven - ermöglichen eine Spontanitätslage, die imstande ist, bemerkenswerte Aha-Effekte auszulösen und somit neue Lösungsmöglichkeiten wahrzunehmen.

\section{Resümee}

Die gewonnene Erkenntnis, dass erst die Klarheit über die Andersartigkeit einer Führungskraft ein konstruktives Miteinander im Team ermöglicht, impliziert, dass der Protagonist des Fallbeispiels erfolgreich von einem dichotomen Denken (Führung und Nähe schließen sich gegenseitig aus) zu einem dialektischen Denken (Führung und Nähe können gleichzeitig bestehen) gelang und somit ein komplexes Verständnis von Führung entwickelte. Demnach zeigt dieser Artikel, dass die Anwendung der psychodramatischen Organisationsaufstellung eine äußerst wertvolle Methode darstellt, die einen Blick außerhalb der üblichen Wahrnehmung wagt. Mit ihren vielfältigen Facetten erweitert die psychodramatische Organisationsaufstellung unseren Blickwinkel und eröffnet uns differenziertere Perspektiven. Das impliziert nicht, dass wir „die Wahrheit“" sehen und abbilden können, aber es hilft, sich interessante Fragen zu stellen und neugierig auf die Komplexität und Vielfalt unserer Welt zu bleiben.

Funding Open access funding provided by Johannes Kepler University Linz.

Open Access Dieser Artikel wird unter der Creative Commons Namensnennung 4.0 International Lizenz veröffentlicht, welche die Nutzung, Vervielfältigung, Bearbeitung, Verbreitung und Wiedergabe in jeglichem Medium und Format erlaubt, sofern Sie den/die ursprünglichen Autor(en) und die Quelle ordnungsgemäß nennen, einen Link zur Creative Commons Lizenz beifügen und angeben, ob Änderungen vorgenommen wurden.

Die in diesem Artikel enthaltenen Bilder und sonstiges Drittmaterial unterliegen ebenfalls der genannten Creative Commons Lizenz, sofern sich aus der Abbildungslegende nichts anderes ergibt. Sofern das betreffende Material nicht unter der genannten Creative Commons Lizenz steht und die betreffende Handlung nicht nach gesetzlichen Vorschriften erlaubt ist, ist für die oben aufgeführten Weiterverwendungen des Materials die Einwilligung des jeweiligen Rechteinhabers einzuholen.

Weitere Details zur Lizenz entnehmen Sie bitte der Lizenzinformation auf http://creativecommons.org/ licenses/by/4.0/deed.de. 


\section{Literatur}

Alvesson, M., \& Jonsson, A. (2018). The bumpy road to exercising leadership: Fragmentations in meaning and practice. Leadership, 14(1), 40-57.

Buer, F. (2003). Aufstellungsarbeit in Organisationen - der klassische Ansatz nach Moreno. Supervision. Mensch Arbeit Organisation, 2, 42-54.

Collinson, D. (2014). Dichotomies, dialectics and dilemmas: new directions for critical leadership studies? Leadership, 10(1), 36-55.

Collinson, D., Smolović Jones, O., \& Grint, K. (2018). "No more heroes": critical perspectives on leadership romanticism. Organization Studies, 39(11), 1625-1647.

Day, D. V., \& Harrison, M. M. (2007). A multilevel, identity-based approach to leadership development. Human Resource Management Review, 17(4), 360-373.

Hammond, M., Clapp-Smith, R., \& Palanski, M. (2017). Beyond (just) the workplace: a theory of leader development across multiple domains. Academy of Management Review, 42(3), 481-498.

Müller, B., \& Schweiger, S. (2018). Führungsbilder, Fallen und HeldInnen. Führungsbilder und die damit verbundenen Erwartungen von und an Führungskräfte. Austrian Management Review, 8, 52-61.

Riepl, R. (2016). Gruppen in Bewegung setzen. Das Wechselspiel zwischen Managementthema und Teamdynamik in der organisationsinternen Aufstellungsarbeit. In G. Weber \& C. Rosselet (Hrsg.), Organisationsaufstellungen. Grundlagen, Settings, Anwendungsfelder (S. 196-214). Heidelberg: Carl-Auer.

Schweiger, S. (2019). Work group effectiveness. Promoting and hindering dynamics. Dissertation,. Linz: Universität Linz.

Schweiger, S., Müller, B., \& Güttel, W. (2020). Barriers to leadership development: why is it so difficult to abandon the hero? Leadership, 16(4), 411-433.

Von Ameln, F., \& Kramer, J. (2007). Organisationen in Bewegung bringen. Handlungsorientierte Methoden für die Personal-, Team- und Organisationsentwicklung. Heidelberg: Springer.

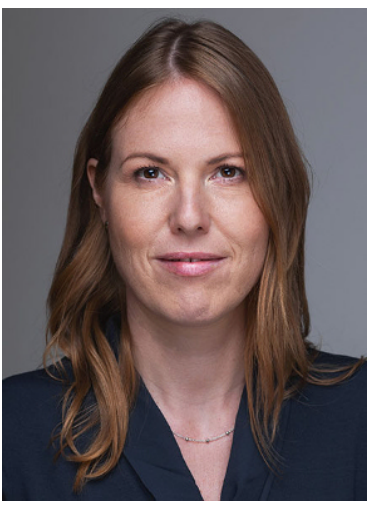

Sylvia Schweiger 1983, Doktorin der Sozial- und Wirtschaftswissenschaften, Lehrbeauftragte an der Universität Linz und Pädagogischen Hochschule Wien, Organisationsberaterin, Psychodramatische Organisationsaufstellerin, Coach nach systemischer Grundhaltung, Supervisorin, Mitglied beim ÖAGG. 